\title{
Synthetic Cannabinoids and Dysphonia: A Case Report
}

Raythatha ${ }^{1}$, Avani BS ${ }^{1}$, Asim Shah ${ }^{2}$, Veronica Tucci ${ }^{3}$ and Nidal Moukaddam ${ }^{*}$

${ }^{1}$ Baylor College of Medicine, USA

${ }^{2}$ Department of Psychiatry \& Behavioral Sciences, Baylor College of Medicine, USA

${ }^{3}$ Section of Emergency Medicine, Baylor College of Medicine, USA

*Corresponding author: Nidal Moukaddam, Baylor College of Medicine, Department of Psychiatry \& Behavioral Sciences, USA, Tel: 713-873-4901; E-mail: nidalm@bcm.edu

Rec date: Jan 01, 2016, Acc date: Jan 08, 2016, Pub date: Jan 18, 2016

Copyright: ( 2016 Raythatha, et al. This is an open-access article distributed under the terms of the Creative Commons Attribution License, which permits unrestricted use, distribution, and reproduction in any medium, provided the original author and source are credited.

\section{Abstract}

Synthetic cannabinoids (SC) have been increasing in popularity throughout the past decade, and are now mainstream drugs of abuse. Undetectable by many urine drug screens, SC are a heterogeneous group of chemicals with various documented side effects including myocardial infarctions, tachycardia, agitation, psychosis, nausea, and vomiting.

Methods: In this case report, we present a 38 year-old female with dysphonia secondary to SC. Our patient developed dysphonia after 2.5 years of regular SC use. She was thoroughly evaluated by her primary care physician and referred to both, otorhinolaryngology and pulmonology, with an exhaustively negative workup. Her dysphonia persisted for 13 months and only improved after she abstained from using SC.

Conclusions and Significance: We could find no previous cases of dysphonia or hoarseness attributed t SC use/ abuse in the literature and is yet another kind of health sequelae frontline providers should look for in chronic users of SC. The previously unrecognized relationship between SC and dysphonia demonstrates our limited understanding of the chemicals' adverse effects. Just as nicotine affects the larynx and causes dysphonia and laryngeal carcinoma, this case also raises the question of whether the chemical composition of SC affects the larynx in the same way and its use leads to an increased risk of laryngeal cancer for users/abusers.
\end{abstract}

Keywords: Synthetic cannabinoids; Dysphonia; Hoarseness; Laryngeal cancer

\section{Introduction}

Over the past decade, synthetic cannabinoids (SC) have been growing in popularity around the world. Also known as "K2", "spice", or "kush", SC are full agonists of cannabinoid receptors (CB); their effect is more pronounced than natural cannabis because the active ingredient of cannabis tetrahydrocannabinol (THC), is only a weak agonist at $\mathrm{CB} 1$.

SC was placed on Schedule I of the Controlled Substances Act in 2011 and the Synthetic Drug Abuse Prevention Act was signed into law in July of 2012. These drugs are marketed in colorful packages and sold in retail stores as incense, bypassing legal systems with the warning "not for human consumption". Many urine drug screens cannot detect SC, and more than 400 varieties have reported/found so far.

These characteristics make the drug widely popular in the teenage and young adult populations. In an anonymous online survey conducted in 2011 resulting in 14,966 participants with a median age of 26 years, 2513 responders or $17 \%$ reported use of SC. Of those participants, 980 responders used SC within the last 12 months and 93 preferred SC over cannabis.

Known side effects of SC include myocardial infarctions, tachycardia, agitation, paranoia, delusions, hallucinations, seizures, nausea, and vomiting. In this case report, we present dysphonia as a side effect of SC, a previously unreported relationship. This case is of importance as it highlights our limited knowledge of the possible health sequelae of SC.

\section{Case Report}

The patient is a 38 year-old Hispanic female with a past medical history significant for bipolar disorder, asthma, and tobacco use who originally presented to the clinic with a five-week history of hoarseness, cough, and xerostomia. The patient had a 23 pack-year history of smoking tobacco and a 2.5 year history of smoking SC through an unfiltered pipe. She had asthma as a child that required inhaler use, but stopped using medication at the age of 10 . The patient also reported an allergy to cats but despite this allergy, lived living with 7 cats in her house.

On initial evaluation by otorhinolaryngology (ENT), she was diagnosed with chronic laryngitis secondary to tobacco use. A flexible fiberoptic laryngoscopy showed leukoplakia, edema, and ulceration of the larynx but no masses. She was advised to discontinue smoking and prescribed a proton pump inhibitor (PPI) and tessalon perlels (benzonatate capsules). However, the PPI did not reduce symptoms. Over the next six months, she had three more episodes of hoarseness. A biopsy of her existing lesion showed an ulcer with reactive squamous mucosa. Repeat laryngoscope was negative for new abnormalities. As her symptoms persisted, she was then referred to pulmonology where infectious disease, autoimmune, and allergic workup was initiated. The patient's pulmonary function tests showed no abnormalities. HIV, 
hepatitis, ANA, anti-dsDNA, CCP IgG/IgA antibody, and allergen panels were all negative. Her CBC showed no eosinophils. A trial of certizine did not alleviate her symptoms. The patient was then prescribed bronchodilators and albuterol inhalers, which she elected not to use.

While being evaluated by ENT and pulmonology, the patient was seen concomitantly by psychiatry for individual psychotherapy and chemical dependency group therapy. During these sessions, the patient noted that cessation of SC use tremendously improved the quality of her voice. However, she was only able to remain abstinent for one to three months at a time. She continued to relapse with acute worsening of her voice. About 13 months after her initial presentation to ENT, she stopped using SC. Her voice significantly improved and eventually returned to baseline. Repeat laryngoscope continued to show chronic laryngitis, but showed no recurrence of a vocal fold lesion. She remains symptom-free 13 months after cessation of SC use despite continued daily use of tobacco and intermittent use of cannabis [1-7].

\section{Discussion}

In this case report we discuss SC as a cause of hoarse voice, a previously unreported side effect of the drug. The differential diagnoses for a hoarse voice include vocal cord nodules, malignancy, gastroesophageal reflux, allergies, and nicotine. A hoarse voice can also be due to other illicit drug use, such as cocaine and methamphetamines.

This patient was well evaluated by ENT and pulmonology, with work-up for traditional causes of hoarseness returning negative. Her hoarseness only improved after cessation of SC use This case highlights the need to inquire about SC in patients presenting with a chief complaint of dysphonia, especially as our understanding of the longterm effects of SC remains limited.
Dysphonia has been a well-recognized side effect of nicotine, but not synthetic cannabinoids. Dysphonia is often the initial presentation of laryngeal carcinoma in smokers. It is possible that SC may act by a similar mechanism, with concern that the development of dysphonia puts the patient at an increased risk for laryngeal carcinoma. Given the prevalence of SC use and its accessibility, it is important to better understand the side effects of SC and its impact on human health.

Furthermore, the extent of necessary otorhinolaryngology and pulmonary workup for patients who present with hoarseness in the setting of SC use is still not fully known, but SC misuse should be considered by primary care physicians and generalists when formulating a differential diagnosis for dysphonia.

\section{References}

1. Celofiga A, Koprivsek J, Klavz J (2014) Use of synthetic cannabinoids in patients with psychotic disorders: case series. J Dual Diagn 10: 168-173.

2. Palmer JJ, Acosta P (2015) Synthetic cannabinoid use in a nationally representative sample of US High school seniors. Drug Alcohol Depend 149: 194-202.

3. Winstock A, Barratt M (2013) Synthetic cannabis: A comparison of patterns of use and effect profile with natural cannabis in a large global sample. Drug Alcohol Depend 131: 106-111.

4. Van Amsterdam J, Brunt T, Van den Brink W (2015) The adverse health effects of synthetic cannabinoids with emphasis on psychosis-like effects. J Psychopharmacol 29: 254-263.

5. McKeever RG, Vearrier D, Jacobs D, LaSala G, Okaneku J, et al. (2015) K2 - Not the spice of life; synthetic cannabinoids and ST elevation myocardial infarction: A case report. JMed Toxicology. 11: 129-131.

6. Trottier A, Massoud E, Brown T (2013) A case of hoarseness and vocal cord immobility. CMAJ 185: 1520-1524.

7. Whited C, Dailey S (2015) Evaluaiton of the Dysphonic Patient. In: Function Preservation in Laryngeal Cancer. Otolaryngol Cin N Am. 